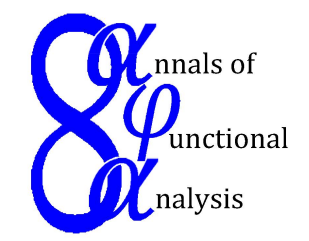

Ann. Funct. Anal. 6 (2015), no. 4, 70-76

http://doi.org/10.15352/afa/06-4-70

ISSN: 2008-8752 (electronic)

http://projecteuclid.org/afa

\title{
LINEAR MAPS PRESERVING ORTHOGONALITY
}

\author{
CHAOQUN CHEN AND FANGYAN LU* \\ Communicated by J. Chmieliński
}

\begin{abstract}
We investigate maps between normed spaces which preserve the orthogonality defined by the norm derivative. These maps are showed to be a scalar multiple of an isometry.
\end{abstract}

\section{INTRODUCTION AND PRELIMINARIES}

Let $X$ be a normed space over $\mathbb{F}$, where $\mathbb{F}$ is the real field $\mathbb{R}$ or the complex field $\mathbb{C}$. For $x$ and $y$ in $X$, we say that $x$ is $B$-orthogonal to $y$, denoted by $x \perp_{B} y$, if $\|x+\lambda y\| \geq\|x\|$ for all $\lambda \in \mathbb{F}$. It is easy to verify that in inner spaces this orthogonality is equivalent to the usual notion of orthogonality, but in general normed spaces is neither symmetric nor additive. However it is homogeneous, thus $x \perp_{B} y$ if and only if $\lambda x \perp_{B} \mu y$ for all nonzero scalars $\lambda, \mu \in \mathbb{F}$. It is well known that a linear map between inner spaces that preserves orthogonality must be a scalar multiple of an isometry.

In [6], Koldobsky proved that a linear map between real normed spaces that preserves $B$-orthogonality must be a scalar multiple of an isometry. Blanco and Turnšek [3] extended this result to complex spaces. Recently, similar investigations have been carried out in normed spaces for sesquilinear form in [9]. Moreover, the approximate orthogonality preserving mappings have been considered in [4].

Besides the $B$-orthogonality, one can define other orthogonality relations in a normed space $X$. Consider two mappings $\rho_{+}^{\prime}, \rho_{-}^{\prime}: X \times X \rightarrow \mathbb{R}$ :

$$
\rho_{ \pm}^{\prime}(x, y)=\lim _{t \rightarrow 0^{ \pm}} \frac{\|x+t y\|^{2}-\|x\|^{2}}{2 t}=\|x\| \lim _{t \rightarrow 0^{ \pm}} \frac{\|x+t y\|-\|x\|}{t}
$$

Date: Received: Jan 4, 2015; Revised: Feb. 17, 2015; Accepted: Mar. 3, 2015.

* Corresponding author.

2010 Mathematics Subject Classification. Primary 46B20; Secondary 47B99.

Key words and phrases. Normed space, orthogonality, map preserving orthogonality. 
which are called norm derivatives [1]. It is easy to show that $\rho_{ \pm}^{\prime}(x, y)=\langle x, y\rangle$ in a real inner space. Thus it is natural to define the following orthogonality relations.

Definition 1.1. Let $X$ be a normed space and $x, y \in X$.

(1). $x$ is $\rho_{+}$-orthogonal to $y$, denoted by $x \perp_{\rho_{+}} y$, if $\rho_{+}^{\prime}(x, y)=0$;

(2). $x$ is $\rho_{-}$-orthogonal to $y$, denoted by $x \perp_{\rho_{-}} y$, if $\rho_{-}^{\prime}(x, y)=0$;

(3). $x$ is $\rho$-orthogonal to $y$, denoted by $x \perp_{\rho} y$, if $\rho_{+}^{\prime}(x, y)+\rho_{-}^{\prime}(x, y)=0$.

In [5], Chmieliński and Wójcik proved that a linear map between real normed spaces that preserves $\rho_{ \pm}$-orthogonality is a scalar multiple of an isometry. In [8], Wójcik proved the same result for maps preserving $\rho$-orthogonality.

The first aim of this paper is to extend results of $[5,8]$ to complex spaces. The second is to define and study a new orthogonality type - $\rho_{*}$-orthogonality.

Definition 1.2. Let $X$ be a normed space and $x, y \in X$. We say that $x$ is $\rho_{*}$-orthogonal to $y$, denoted by $x \perp_{\rho_{*}} y$, if $\rho_{+}^{\prime}(x, y) \rho_{-}^{\prime}(x, y)=0$.

We will show that a linear map preserving $\rho_{*}$-orthogonality between normed spaces is a scalar multiple of an isometry.

We close this section with some preliminaries. First, we collect some properties of norm derivatives, which can be found, for example, in $[1,2]$.

Proposition 1.3. Let $x$ and $y$ be in a normed space $X$.

(1). $\rho_{-}^{\prime}(x, y) \leq \rho_{+}^{\prime}(x, y)$;

(2). $\rho_{-}^{\prime}(x, y)=-\rho_{+}^{\prime}(x,-y)=-\rho_{+}^{\prime}(-x, y)$;

(3). $\rho_{ \pm}^{\prime}(x, x)=\|x\|^{2}$;

(4). $\rho_{ \pm}^{\prime}(\alpha x, \beta y)=|\alpha \beta| \rho_{ \pm}^{\prime}\left(x, e^{i(\theta-\omega)} y\right), \quad \alpha=|\alpha| e^{i \omega}, \beta=|\beta| e^{i \theta}$;

(5). $\rho_{ \pm}^{\prime}(x, \alpha x+y)=\operatorname{Re} \alpha\|x\|^{2}+\rho_{ \pm}^{\prime}(x, y)$ for $\alpha \in \mathbb{F}$;

(6). $\left|\rho_{ \pm}^{\prime}(x, y)\right| \leq\|x\|\|y\|$.

Recall that a support functional $\phi$ at $x \in X$ is a norm-one linear functional in $X^{*}$ such that $\phi(x)=\|x\|$. By the Hahn-Banach Theorem, there always exists at least one such functional for every $x \in X$. Recall also that $X$ is smooth at the point $x$ in $X$ if there exists a unique support functional at $x$. From [2], for $x, y \in X, x \neq 0, \rho_{+}^{\prime}(x, y)=\|x\| \sup \{\operatorname{Re} \varphi(y): \varphi \in S(x)\}$ and $\rho_{-}^{\prime}(x, y)=$ $\|x\| \inf \{\operatorname{Re} \varphi(y): \varphi \in S(x)\}$, where $S(x)$ is the set of all support functionals at $x$. Thus, $X$ is smooth at $x$ if and only if $\rho_{+}^{\prime}(x, y)=\rho_{-}^{\prime}(x, y)$ for all $y \in X$.

It turns out that the smoothness is closely related to the Gateaux differentiability. Recall that the norm $\|\cdot\|$ is said to be Gateaux differential at $x \in X$ if the limit

$$
f_{x}(y)=\lim _{t \rightarrow 0} \frac{\|x+t y\|-\|x\|}{t}
$$

exists for all $y \in X$. We call such $f_{x}$ the Gateaux differential at $x$ of $\|\cdot\|$. It is not difficult to verify that $f_{x}$ is a bounded real linear functional on $X$. When $x$ is a smooth point, it is easy to see that $\rho_{+}^{\prime}(x, y)=\rho_{-}^{\prime}(x, y)=\|x\| f_{x}(y)$ for $y \in X$. Therefore $X$ is smooth at $x$ if and only if the norm is Gateaux differential at $x$.

Proposition 1.4. [7] Every norm on $\mathbb{R}^{n}$ is Gateaux differentiable $\mu^{n}$-a.e. on $\mathbb{R}^{n}$. Here, $\mu^{n}$ is the Lebesgue measure on $\mathbb{R}^{n}$. 


\section{LiNEAR MAPS PRESERVING $\rho_{+}, \rho_{-}, \rho_{*}$-ORTHOGONALITY}

In this section, we characterize linear maps of normed spaces which preserve $\rho_{*^{-}}$ orthogonality ( $\rho_{+}$-orthogonality, $\rho_{-}$-orthogonality). Recall that $x$ is $\rho_{*}$-orthogonal to $y$ if $\rho_{+}^{\prime}(x, y)=0$ or $\rho_{-}^{\prime}(x, y)=0$. We need a lemma which characterizes the $B$-orthogonality using norm derivatives.

Lemma 2.1. Let $x$ and $y$ be in a normed space $X$. Then the following conditions are equivalent.

(1). $x \perp_{B} y$;

(2). $\rho_{-}^{\prime}(x, \lambda y) \leq 0$ for all $\lambda \in \mathbb{F}$;

(3). $\rho_{+}^{\prime}(x, \lambda y) \geq 0$ for all $\lambda \in \mathbb{F}$.

Proof. $(1) \Rightarrow(2)$. Let $\lambda$ be in $\mathbb{F}$. Since $x \perp_{B} y$, it follows that $\|x+t \lambda y\|-\|x\| \geq 0$ and then $\frac{\|x+t \lambda y\|-\|x\|}{t} \leq 0$ for all $t<0$. So

$$
\rho_{-}^{\prime}(x, \lambda y)=\lim _{t \rightarrow 0^{-}} \frac{\|x+t \lambda y\|-\|x\|}{t} \leq 0 .
$$

$(2) \Rightarrow(3)$. Let $\lambda$ be in $\mathbb{F}$. Then $-\lambda \in \mathbb{F}$. By the condition $(2), \rho_{-}^{\prime}(x,-\lambda y) \leq 0$. Hence, by Proposition 1.3, $\rho_{+}^{\prime}(x, \lambda y)=-\rho_{-}^{\prime}(x,-\lambda y) \geq 0$.

$(3) \Rightarrow(1)$. Let $\lambda$ be in $\mathbb{F}$. By the condition $(3), \rho_{+}^{\prime}(x, \lambda y) \geq 0$. Hence by Proposition 1.3,

$$
\|x\|^{2} \leq\|x\|^{2}+\rho_{+}^{\prime}(x, \lambda y)=\rho_{+}^{\prime}(x, x+\lambda y) \leq\|x\|\|x+\lambda y\| .
$$

This implies that $\|x\| \leq\|x+\lambda y\|$ for all $\lambda \in \mathbb{F}$. Namely, $x \perp_{B} y$.

The main result in this section is as follows.

Theorem 2.2. Let $X, Y$ be normed spaces, $T: X \rightarrow Y$ a nonzero, linear map. Then the following conditions are equivalent.

(1). T preserves $\rho_{*}$-orthogonality;

(2). T preserves $\rho_{+}$-orthogonality;

(3). $T$ preserves $\rho_{-}$-orthogonality;

(4). T preserves $B$-orthogonality;

(5). $\|T x\|=\|T\|\|x\|$ for all $x \in X$.

Proof. That (5) implies (1)-(4) is obvious. From [3], (4) implies (5). Now we show that each of conditions (1)-(3) implies (4).

First suppose that (1) holds. Let $x, y \in X, x \neq 0$ and suppose that $x \perp_{B} y$. Our task is to show that $T x \perp_{B} T y$. By Lemma 2.1, this is equivalent to show that $\rho_{+}^{\prime}(T x, \lambda T y) \geq 0$ for all $\lambda \in \mathbb{F}$.

By Proposition 1.3, we have, for $\lambda \in \mathbb{F}$, that

$$
\rho_{+}^{\prime}\left(x,-\frac{\rho_{+}^{\prime}(x, \lambda y)}{\|x\|^{2}} x+\lambda y\right)=-\frac{\rho_{+}^{\prime}(x, \lambda y)}{\|x\|^{2}}\|x\|^{2}+\rho_{+}^{\prime}(x, \lambda y)=0 .
$$

Since $T$ preserves $\rho_{*}$-orthogonality, it follows that $\rho_{+}^{\prime}\left(T x,-\frac{\rho_{+}^{\prime}(x, \lambda y)}{\|x\|^{2}} T x+\lambda T y\right)=$ 0 or $\rho_{-}^{\prime}\left(T x,-\frac{\rho_{+}^{\prime}(x, \lambda y)}{\|x\|^{2}} T x+\lambda T y\right)=0$. Hence by Proposition 1.3 and Lemma 
2.1, we have, for $\lambda \in \mathbb{F}$, that

or

$$
\rho_{+}^{\prime}(T x, \lambda T y)=\frac{\|T x\|^{2}}{\|x\|^{2}} \rho_{+}^{\prime}(x, \lambda y) \geq 0,
$$

$$
\rho_{+}^{\prime}(T x, \lambda T y) \geq \rho_{-}^{\prime}(T x, \lambda T y)=\frac{\|T x\|^{2}}{\|x\|^{2}} \rho_{+}^{\prime}(x, \lambda y) \geq 0 .
$$

Consequently, we have that $\rho_{+}^{\prime}(T x, \lambda T y) \geq 0$ for all $\lambda \in \mathbb{F}$.

In a similar way, we can show that (2) implies (4).

Finally, using $\rho_{-}^{\prime}\left(x,-\frac{\rho_{-}^{\prime}(x, \lambda y)}{\|x\|^{2}} x+\lambda y\right)=0$, we can show that $(3)$ implies (4).

The proof is complete.

\section{LINEAR MAPS PRESERVING $\rho$-ORTHOGONALITY}

In this section, we characterize linear maps of normed spaces which preserve $\rho$-orthogonality in the complex space case. We need a lemma.

Lemma 3.1. [3] Let $\|\cdot\|$ be any norm on $\mathbb{F}^{2}$ and let $D \subseteq \mathbb{F}^{2}$ be a set of all non-smooth points. Then there exists a path $\gamma:[0,2] \rightarrow \mathbb{F}^{2}$ of the form:

$$
\gamma(t):= \begin{cases}(1, t \xi), & t \in[0,1] \\ (1,(2-t) \xi+(t-1)), & t \in[1,2],\end{cases}
$$

for some $\xi \in \mathbb{F}, \xi \neq 0,1$, so that $\nu\{t: \gamma(t) \in D\}=0$.

The main result is as follows.

Theorem 3.2. Let $X, Y$ be normed spaces, $T: X \rightarrow Y$ a nonzero, linear map. Then $T$ preserves $\rho$-orthogonality if and only if $T$ is a scalar multiple of an isometry.

Proof. The sufficiency is obvious. We now suppose that $T$ preserves $\rho$-orthogonality and want to prove that $T$ is a scalar multiple of an isometry. For convenience, we write $\rho^{\prime}=\frac{1}{2}\left(\rho_{+}^{\prime}+\rho_{-}^{\prime}\right)$. Then $x \perp_{\rho} y$ if and only if $\rho^{\prime}(x, y)=0$.

Claim 1. $T$ is injective.

Suppose on the contrary that $T x=0$ for some non-zero $x \in X$. Let $y$ be a vector in $X$ which is independent of $x$. Then we can choose a non-zero $\beta \in \mathbb{R}$ such that $0<\frac{\beta\|y\|}{\|x+\beta y\|}<1$. Let $z=x+\beta y$. A computation shows $\rho^{\prime}\left(z,-\frac{\rho^{\prime}(z, y)}{\|z\|^{2}} z+y\right)=0$. Since $T$ preserves $\rho$-orthogonality, it follows that $\rho^{\prime}\left(T z,-\frac{\rho^{\prime}(z, y)}{\|z\|^{2}} T z+T y\right)=0$. Since $T x=0$, by Proposition 1.3 we have that

$$
\begin{aligned}
0 & =\rho^{\prime}\left(T z,-\frac{\rho^{\prime}(z, y)}{\|z\|^{2}} T z+T y\right) \\
& =\rho^{\prime}\left(\beta T y,\left(1-\frac{\rho^{\prime}(z, y)}{\|z\|^{2}} \beta\right) T y\right)=\beta\left(1-\frac{\rho^{\prime}(z, y)}{\|z\|^{2}} \beta\right)\|T y\|^{2} .
\end{aligned}
$$


By Proposition 1.3,

$$
\left|\frac{\rho^{\prime}(z, y)}{\|z\|^{2}} \beta\right| \leq \frac{\|z\|\|y\| \beta}{\|z\|^{2}}=\frac{\beta\|y\|}{\|x+\beta y\|}<1 .
$$

So $1-\frac{\rho^{\prime}(z, y)}{\|z\|^{2}} \beta \neq 0$. This together with (3.1) yields that $T y=0$ for all $y$ independent of $x$. Hence $T=0$, a contradiction.

Claim 2. Let $x$ and $y$ be in $X$ and suppose that $\|x\|=\|y\|$. Then $\|T x\|=\|T y\|$.

If $x$ and $y$ are linearly dependent, saying $y=\mu x$ for some $\mu \in \mathbb{F}$, then $|\mu|=1$, and $\|T y\|=\|\mu T x\|=\|T x\|$. Now let us suppose that $x$ and $y$ are linearly independent. Let $M$ be the linear subspace spanned by $x$ and $y$. For $u \in M$, define $\|u\|_{T}=\|T u\|$. Then by Claim $1,\|\cdot\|_{T}$ is a norm on $M$. Clearly, $\left(M,\|\cdot\|_{T}\right)$ is smooth at $u$ if and only if $T M$ is smooth at $T u$. Let $\Delta$ be the set of all those $u \in M$ at which at least one of the norms, $\|\cdot\|$ or $\|\cdot\|_{T}$, is not Gateaux differentiable.

For $u \in M \backslash \Delta$, by $f_{u}$ and $g_{u}$ denote the Gateaux differentials at $u$ of $\|\cdot\|$ and $\|\cdot\|_{T}$ on $M$ respectively. Let $v \in \operatorname{ker} f_{u}$. Then $\rho^{\prime}(u, v)=\|u\| f_{u}(v)=0$ since $(M,\|\cdot\|)$ is smooth at $u$. Hence $\rho^{\prime}(T u, T v)=0$ since $T$ preserves $\rho$-orthogonality. Moreover, since $(M,\|\cdot\|)$ is smooth at $u$, we have $g_{u}(v)=\frac{1}{\|T u\|} \rho^{\prime}(T u, T v)=0$. So, we have ker $f_{u} \subseteq \operatorname{ker} g_{u}$ for all $u \in M \backslash \Delta$, equivalently, there exists a function $\lambda: M \backslash \Delta \rightarrow \mathbb{C}$ such that $g_{u}=\lambda(u) f_{u}$ for all $u \in M \backslash \Delta$. As

$$
\|T u\|=g_{u}(u)=\lambda(u) f_{u}(u)=\lambda(u)\|u\|, u \in M \backslash \Delta,
$$

it is easily seen that $\lambda$ is in fact real valued.

Let $L: \mathbb{C}^{2} \rightarrow M,(\alpha, \beta) \mapsto \alpha x+\beta(y-x)$. Clearly, $L$ is a linear isomorphism. Set $D=L^{-1}(\Delta)$, then $D$ is the set of those points $(\alpha, \beta) \in \mathbb{C}^{2}$ at which at least one of the functions $(\alpha, \beta) \mapsto\|L(\alpha, \beta)\|$ or $(\alpha, \beta) \mapsto\|L(\alpha, \beta)\|_{T}$ is not Gateaux differentiable. Both these functions are norms in $\mathbb{C}^{2}=\mathbb{R}^{4}$, hence by Proposition $1.4, \nu^{4}(D)=0$. Let $\gamma:[0,2] \rightarrow \mathbb{C}^{2}$ be the path obtained in Lemma 3.1. Then $\Gamma:[0,2] \rightarrow M$ defined by

$$
\Gamma(t)=\frac{\|x\|}{\|L \gamma(t)\|} L \gamma(t), t \in[0,2]
$$

is a path from $x$ to $y$ such that $\|\Gamma(t)\|=\|x\|$ and $\nu\{t: \Gamma(t) \in \Delta\}=\nu\{t: \gamma(t) \in$ $D\}=0$. Note that $t \mapsto\|L \gamma(t)\|$ and $t \mapsto\|L \gamma(t)\|_{T}$ are Lipschitz functions and , therefore, absolutely continuous. Indeed,

$$
L \gamma(t)= \begin{cases}x+t \xi(y-x), & t \in[0,1] \\ x+((2-t) \xi+(t-1))(y-x), & t \in[1,2]\end{cases}
$$

Hence

(a) if $t_{1}, t_{2} \in[0,1]$, then

$$
\left\|L \gamma\left(t_{1}\right)\right\|-\left\|L \gamma\left(t_{2}\right)\right\| \leq\left\|L \gamma\left(t_{1}\right)-L \gamma\left(t_{2}\right)\right\|=\left|t_{1}-t_{2}\right||\xi|\|y-x\| ;
$$

(b) if $t_{1}, t_{2} \in[1,2]$, then

$$
\left\|L \gamma\left(t_{1}\right)\right\|-\left\|L \gamma\left(t_{2}\right)\right\| \leq\left\|L \gamma\left(t_{1}\right)-L \gamma\left(t_{2}\right)\right\|=\left|t_{1}-t_{2}\|1-\xi \mid\| y-x \| ;\right.
$$


(c) if $t_{1} \in[0,1], t_{2} \in[1,2]$, then

$$
\begin{aligned}
\left|\left\|L \gamma\left(t_{1}\right)\right\|-\left\|L \gamma\left(t_{2}\right)\right\|\right| & \leq\left\|L \gamma\left(t_{1}\right)-L \gamma\left(t_{2}\right)\right\| \\
& =\|y-x\|\left|\left(t_{1}+t_{2}-2\right) \xi-\left(t_{2}-1\right)\right| \\
& \leq\|y-x\|\left(\left|\left(t_{1}+t_{2}-2\right) \xi\right|+\left|t_{2}-1\right|\right) \\
& \leq\|y-x\|\left(\left|t_{1}-t_{2}\right||\xi|+\left|t_{1}-t_{2}\right|\right) \\
& =\left|t_{1}-t_{2}\right|(1+|\xi|)\|y-x\| .
\end{aligned}
$$

So $t \mapsto\|L \gamma(t)\|$ satisfies Lipschitz conditions. Similarly, $t \mapsto\|L \gamma(t)\|_{T}$ satisfies Lipschitz conditions. It follows that

$$
\|\Gamma(t)\|_{T}=\frac{\|x\|\|L \gamma(t)\|_{T}}{\|L \gamma(t)\|}
$$

is absolutely continuous too, and that

$$
\nu\left\{t: \Gamma^{\prime}(t) \text { does not exist }\right\}=\nu\left\{t:\|L \gamma(t)\|^{\prime} \text { does not exist }\right\}=0 .
$$

Since $t \mapsto\|\Gamma(t)\|=\|x\|$ is a constant function, we have

$$
\begin{aligned}
\|\Gamma(t)\|_{T}^{\prime} & =\lim _{\Delta t \rightarrow 0} \frac{\|\Gamma(t+\Delta t)\|_{T}-\|\Gamma(t)\|_{T}}{\Delta t} \\
& =\lim _{\Delta t \rightarrow 0} \frac{\left\|\Gamma(t)+\Delta t \Gamma^{\prime}(t)\right\|_{T}-\|\Gamma(t)\|_{T}}{\Delta t} \\
& =g_{\Gamma(t)}\left(\Gamma^{\prime}(t)\right)=\lambda(\Gamma(t)) f_{\Gamma(t)}\left(\Gamma^{\prime}(t)\right) \\
& =\lambda(\Gamma(t))\|\Gamma(t)\|^{\prime}=0
\end{aligned}
$$

$\nu$-a.e. on $[0,2]$. Hence $t \mapsto\|\Gamma(t)\|_{T}$ is a constant function and we have that $\|x\|_{T}=\|\Gamma(0)\|_{T}=\|\Gamma(2)\|_{T}=\|y\|_{T}$, i.e., $\|T x\|=\|T y\|$. Claim 2 is established.

Now we fix a unit vector $x_{0}$ in $X$. For a non-zero vector $x \in X$, by Claim 2, we have that $\left\|T\left(\frac{1}{\|x\|}\right) x\right\|=\left\|T x_{0}\right\|$. Therefore $\|T x\|=\left\|T x_{0}\right\|\|x\|$ for all $x \in X$. This implies that $\frac{1}{\left\|T x_{0}\right\|} T$ is an isometry.

\section{REFERENCES}

1. C. Alsina, J. Sikorska and M. Santos Tomás, Norm Derivatives and Characterizations of Inner Product Spaces, World Scientific, Hackensack, NJ, 2009.

2. A. Bachir and A. Segres, Numerical range and orthogonality in normed spaces, Filomat 23 (2009), 21-41.

3. A. Blanco and A. Turnšek, On maps that preserve orthogonality in normed spaces, Proc. Roy. Soc. Edinburgh Sect. A 136 (2006), 709-716.

4. J. Chmieliński, Linear mappings approximately preserving orthogonality, J. Math. Anal. Appl. 304 (2005), 158-169.

5. J. Chmieliński and P. Wójcik, On a $\rho$-orthogonality, Aequationes Math. 80 (2010), 45-55.

6. A. Koldobsky, Operators preserving orthogonality are isometries, Proc. Roy. Soc. Edinburgh Sect. 123A (1993), 835-837.

7. R. Phelps, Convex functions, monotone operators and differentiability, Second edition, Lecture Notes in Mathematics 1364, Springer,1993.

8. P. Wójcik, Linear mappings preseving $\rho$-orthogonality, J. Math. Anal. Appl. 386 (2012), $171-176$. 
9. P. Wójcik, Operators preserving sesquilinear form, Linear Algebra Appl. 469 (2015), 531538.

Department of Mathematics, Soochow University, Suzhou 215006, P. R. China. E-mail address: ccywolf@163.com

E-mail address: fylu@suda.edu.cn 